\title{
Can Orbital Clustering of KBOs in the Ecliptic be due to the Solar Toroidal Field Generated Spacetime Dragging?
}

\author{
Babur M. Mirza \\ Department of Mathematics, \\ Quaid-i-Azam University, Islamabad. 45320. PK.
}

March 2, 2020

\begin{abstract}
The Kuiper belt objects (KBOs) exhibit an orbital clustering of the outer planets lying at perihelion distances larger than Neptune and semimajor axes greater than $150 \mathrm{AU}$ from the Sun. This implies a hitherto unknown dynamical mechanism to counter randomizing of the orbital elements caused by the giant solar system planets. Using the toroidal field induced frame-dragging we deduce here the observed range of the Kuiper belt region, the semi-major axis of Sedna like objects in the Kuiper belt, as well as the orbital clustering of the KBOs in the ecliptic, without assuming dynamical effects induced by trans-Neptunian-objects (TNOs). We also calculate the orbital precession rates for the inner planets and show their correspondence, within the range of observational accuracy, with recent planetary ephemerides.
\end{abstract}

\section{Introduction}

The Kuiper belt objects (KBOs) span a broad range of perihelion distances, ranging approximately from $35 \mathrm{AU}$ to $80 \mathrm{AU}$. While some KBOs interact much more strongly with Neptune, distant KBOs exhibit relatively stable orbits not affected by Neptune's gravity. In the Kuiper belt, weak clustering of objects undergo a rapid dynamical chaos which leaves little orbital structure in the distant belt region (Batygin \& Brown 2016; Marcos \& Marcos 2014). The more stable orbits, however, may still disperse due to the precession induced by giant planets, including Jupiter, Saturn, Uranus, and Neptune. Numerical simulations (Madigan \& McCourt 2015; Trujillo \& Sheppard 2014; Batygin, Brown, \& Fraser 2011; Levison et al. 2008) of the orbital dynamics of the distant KBOs show Sedna's orbital precession by $0.15 \mathrm{deg} / \mathrm{Myrs}$, and 2014 SR349 precession at 0.8 $\mathrm{deg} / \mathrm{Myr}$. This range of precession rates leads to a far too shorter time for the KBOs' orbits to disband, in approximately 100Myr. The observed alignment 
of the orbital semi-major axis at a distance nearly $250 \mathrm{AU}$ implies a hitherto unknown dynamical mechanism, other than the secular perturbation induced by the major planets inside the solar system.

In orbital dynamics of the KBOs, general relativistic effects, such as the Lense-Thirring frame dragging of spacetime (Lense \& Thirring 1918; Rindler 1997; Tartaglia 2002; Iorio 2010), can be in general ruled out owing to their extremely small magnitude. On the other hand, for inner planets, it has been shown (Iorio 2018; Iorio 2012) that the Lense-Thirring frame dragging effect has measurable magnitude which lie at the same or above the observational error. It has also been used in detailed modelling of the planetary dynamics in the solar system 11. The on-going efforts to measure the relativistic frame-dragging effect near the Earth, include high precision artificial satellite measurements (Lucchesi et.al. 2019; Renzetti 2013). On the other hand long range gravitational effects, such as those exhibited in the orbital dynamics of the KBOs, cannot be accounted for by typical general relativistic effects. In this case the dragging induced by the giant planets, and even that due to the Sun, is negligible. Moreover, the metastable/stable orbital dynamics of KBOs imply a cut-off region, where as the effective potential due to the Lense-Thirring frame dragging decays as inverse cube of the orbital distance. Similarly, the modification induced by the solar Lense-Thirring frame dragging can be ruled out as significant in the dynamics of KBOs in view of the clustering of the KBOs in the ecliptic. Within the Newtonian dynamics, the clustering of KBOs orbits, can however be modelled under the postulate of a trans-Neptunian planet (Batygin \& Brown 2016a; Batygin \& Brown 2016b; Brown \& Batygin 2016; Batygin et.al. 2019; Iorio 2017). Notably, in these models, the nineth planet (Telisto) causes secular correction to the Hamiltonian which has an additive harmonic form.

Although typical general relativistic effects remain small in the case of dynamics around normal stars, in compact stars spacetime effects play a key role in various energy generation mechanisms. For example, in these systems the wrapping of spacetime, affected by the extremely high magnetic field energy density, plays an important role in accretion dynamics (Mereghetti 2008; Thompson \& Duncan 1995, Harding \& Lai 2006; Makishima 2014; Ciolfi, 2014; Muhlberger et al., 2014; Mitchell et al., 2015). The relativistic field amplification in these stars is up to the order of $10^{12} G$, where as in magnetars the surface currents indicate fields as high as $10^{15} G$ (Tiengo et al. 2013). In such cases the high energy density of the star is modified by the spacetime wrapping. This results in various observable phenomena, such as dragging induced surface currents and high temperature gradients in the stellar atmosphere. Moreover, this indicates that coupling of magnetic field to the background spacetime can be a far more effective mechanism in the stellar dynamics than spacetime induced effects only. Here the magnetic field can not only affect accretion around the star via the Lorentz force, but can also induce modifications in the spacetime itself. The magnetic field energy density acts here as a direct measure of the modification

\footnotetext{
${ }^{1}$ For recent overviews of GR and the challenges faced, see, e,g., (Iorio, 2005; Debono \& Smoot 2016) and references therein.
} 
induced in the background spacetime, since it contributes to the total energy density of the system.

The discovery of the toroidal magnetic field in the Sun and Sun-like stars implies that normal stars also posses analogous conditions, although with relatively weaker fields. In these cases, the toroidal field is largely generated at the stellar surface, and is sustained by differential rotation inside the star (Petit et al. 2008). Comparable in magnitude to the poloidal field, the toroidal field ranges from $1 G$ to $10^{3} G$ during the solar cycle. As in the case of dense stars, the magnetic field energy density can cause modification in the spacetime structure around a normal star like the Sun. For the Sun, the magnetic field directly affects charge accretion via Lorentz force up to the magnetosphere, and has negligible long range effect on dynamics in the solar system. However, outside the solar surface not only the spacetime curvature but also spacetime frame-dragging has effects manifest in the orbital dynamics of the inner planets, such as the relativistic perihelion advance of Mercury. The coupling of magnetic field energy density to the background spacetime can be a more effective means of modifying the orbital dynamics since it can extend spacetime dragging, analogous to the toroidal field energy induced dragging in compact gravitational sources. In these cases, the magnetic field energy density acts as an additional, locally distributed pressure, causing the spacetime dragging effects to amplify. In fast rotating dense stars, like the neutron stars, spacetime wrapping is closely bound to the surface of the star, causing the observed surface currents in these objects. In comparison, for Sun-like gravitational systems, the relatively weaker wrapping can extends much farther in space. The coupling of spacetime wrapping can be viewed here as an increase in the magnetic field lines (per unit volume) in vicinity of the rotating gravitational source. Conversely, the magnetic field energy density causes local stresses in the spacetime structure, thus inducing modifications in the gravitational field of the star. The magnetic field energy density corresponds here directly to the enhancement induced in the spacetime, particularly in the case of the toroidal field coupling around the star. The effect can be significantly high in the equatorial plane of the star where the dragging is maximum, and can cause orbital recession. The coupled field dragging can be more effective in orbital precession than the Lense-Thirring frame-dragging, since it has larger local magnitude and a longer range. In observational tests, the effect of such a long-range frame dragging may therefore be detectable both in the orbital precession of the inner planets as well as in the orbital dynamics of the outer solar system. As shown below, the coupling has the form of a secular perturbation, as postulated for Telisto, and can induce long term cyclic changes in the KBOs' orbits. The spacetime vorticity here counterbalances the gravitational attraction by providing the outward velocity drag, extending at very large distances.

Here we show that the solar toroidal field modification induced in the spacetime frame-dragging exhibits observable secular dynamical trends in the orbital dynamics around the Sun. We thus derive the anomalous shifts in the inner planetary orbits in the solar system and, as a long-range dragging effect, deduce the distance of the Kuiper belt region along with the high eccentricity orbits of 
the KBOs, as well as their observed clustering in the solar ecliptic. In this we do not assume the nineth planet hypothesis, or the existence of extra-solar effects such as the galactic dark matter. The coupling of toroidal field to the spacetime dragging is shown here to be derivable from General Relativistic (GR) Maxwell equations in the next section. Also, it can be shown generally that the modification in the dragging of spacetime is due to the axial symmetry, hence valid for any rotating gravitational source in stable equilibrium, such as that with a Kerr background spacetime. We then deduce the toroidal magnetic field energy density as a function of the Lense-Thirring frequency, and calculate the energy density of the toroidal field. The modified potential is then used to derive the orbital parameters of the KBOs, including the semi-major axis, the drag velocity, radius of the Kuiper belt, and also radius of the solar system. We moreover show that the perturbations induced in the inner planetary orbits correspond to the observed anomalous extraperihelion precessions in inner planetary orbits in the solar system.

\section{Toroidal Solar Field and the Spacetime Frame- Dragging}

The toroidal field induced spacetime dragging can be derived from coupling of the magnetic field to the gravitational field of a massive source. For this we consider the Maxwell equations for the electromagnetic field in a curved spacetime around a rotating mass (Mirza 2017; Pétri 2013; Mirza 2007; Oron 2002). In this case the background spacetime is axially symmetric and, for slowly rotating objects, is given by the linearized Kerr metric,

$$
d s^{2}=-e^{2 \Phi(r)} d t^{2}+e^{-2 \Phi(r)} d r^{2}+d \Omega^{2}-2 \omega(r) r^{2} \sin ^{2} \theta d \varphi d t,
$$

where $d \Omega^{2}=r^{2} d \theta^{2}+r^{2} \sin ^{2} \theta d \varphi^{2}$, and $e^{2 \Phi(r)}=(1-2 M / r)$. Here $\omega(r)$ is the Lense-Thirring frame-dragging frequency, equal to $2 j / r^{3}$, where as $j$ is the angular momentum of the star with mass $M$. Also, the velocity 4-vector compatible with the metric is that of a co-moving observer, given by $u^{\alpha}=$ $\left(e^{-\Phi(r)}, 0,0, \omega e^{-\Phi(r)}\right)$.

General Relativistic Maxwell equations in a curved spacetime are given by (Landau \& Lifshitz 1980),

$$
\begin{gathered}
F_{\alpha \beta, \gamma}+F_{\beta \gamma, \alpha}+F_{\gamma \alpha, \beta}=0, \\
\left(\sqrt{-g} F^{\alpha \beta}\right)_{, \beta}=4 \pi \sqrt{-g} J^{\alpha},
\end{gathered}
$$

where $g$ represents the determinant of the metric tensor $g_{\alpha \beta}$, and $F_{\alpha \beta}$ is the electromagnetic field tensor. It can be shown that the assumption of an everywhere finite $J^{\alpha}$ leads to the condition that in a co-moving frame $E^{\alpha}=0=E_{\alpha}$ (Lichnerowicz 1967). Therefore, for the exterior region, the electromagnetic field tensor takes the form $F_{\alpha \beta}=\sqrt{-g} \epsilon_{\alpha \beta \gamma \delta} u^{\gamma} B^{\delta}$, and in the contravariant components $F^{\alpha \beta}=-(-g)^{-1 / 2} \epsilon^{\alpha \beta \gamma \delta} u_{\gamma} B_{\delta}$, where $\epsilon_{\alpha \beta \gamma \delta}$ is the four index Levi -Civita symbol. 
Maxwell equations (2) and (3) can be solved to give the magnetic field as a function of $(t, r, \theta, \varphi)$. The full set of Maxwell equations forms a system of eight coupled partial differential equations (Mirza 2007). Under the condition that $\omega \neq 0$ and $B_{t} \neq 0$, it can be verified that the magnetic field around the star has the form,

$$
\left(B^{\alpha}\right)=B_{0}\left(0,0, \frac{A(\theta) \sin \theta \sin \xi}{u_{t} r^{2} \sin ^{2} \theta}, \frac{\cos \xi}{u_{t} r^{2} \sin ^{2} \theta}\right),
$$

where $B_{0}$ is a constant, $\xi=\varphi-\omega t$. Also,

$$
A(\theta)=\int \frac{d \theta}{\sin \theta}
$$

\section{Dragging Effect in the Orbits of KBOs and Inner Planetary Orbits}

According to equations (4) and (5), the contribution of the poloidal field to the total field energy density for any close loop for $\theta$ vanishes. However, the toroidal field contributes to the energy density of the surrounding field, for a rotating source, by

$$
\epsilon_{\varphi}=\frac{B_{0}^{2} \cos ^{2} \xi}{u_{t}^{2} r^{2} \sin ^{2} \theta} .
$$

The field energy density is minimum in the equatorial plane $(\theta=\pi / 2)$ and maximum at the poles. This is significant for the orbital clustering, since the toroidal field energy density provides the outward velocity drag around the Sun.

To calculate the velocity drag induced by the solar toroidal field, we consider dragging effects in the equatorial plane $\theta=\pi / 2$ of the Sun. We therefore have for the solar toroidal field,

$$
B_{\varphi}=-\frac{B_{0}}{r \sqrt{1-\frac{R_{s}}{r}}} \cos (\varphi-\omega t),
$$

where $r>R_{s}$, and $R_{s}=2 G M / c^{2}$ is the Schwarzschild radius.

For a slowly rotating star like the Sun, $\omega<<1$, and the cosine factor depends on the choice of the coordinate $\varphi$, hence can be included in the constant $B_{0}$. The radial drag therefore comes from the factor $B_{0} /\left(r \sqrt{1-R_{s} / r}\right)$. Therefore, if $R$ denotes the radius of the disc in the ecliptic (as the equatorial plane of the star), containing a total mass $M$, then the total energy density of the field up to the radius $R$ is given by,

$$
\epsilon=\int_{0}^{R} 4 \pi r^{2} \epsilon_{\varphi} d r
$$

Substituting from equation (7) and integrating by parts we obtain,

$$
\epsilon=4 \pi B_{0}^{2}\left[R+R_{s} \ln \left(R-R_{s}\right)\right] \cos ^{2}(\varphi-\omega(R) t), R>R_{s},
$$


where $\omega^{2}$ and higher order powers have been neglected. We see that this energy contributes to the total energy of the system, hence like the gravitational potential energy, must be independent of the mass of the test body. Also, the second term in equation (9) is very small as compared to the first, and can be neglected. Therefore, the energy density due to the toroidal-gravitational field coupling is,

$$
\epsilon=4 \pi B_{0}^{2} R \cos ^{2}(\varphi-\omega(R) t),
$$

which depends on the frame-dragging frequency $\omega$ cyclically. In general, for gravitationally bound systems with a power law potential function, the virial theorem applies. For potential energy $V(R)$ and kinetic energy $K=v_{d}^{2} / 2$ (per unit mass), we thus have $2 K=V=\epsilon$. Therefore, for the radial velocity drag $v_{d}$, we have,

$$
v_{d}=B_{0} \sqrt{4 \pi R} \cos (\varphi-\omega(R) t)
$$

Equation (11) gives the outward (positive) drag velocity for a test body in the gravitational field of the Sun, which is in addition to its orbital velocity induced by the Newtonian gravitational potential.

Since $\omega<<1$, and $\varphi$ depends on the choice of the orientation of the observer's coordinates, the energy density per unit mass due to toroidal-gravitational field coupling is $\epsilon=4 \pi B_{0}^{2} R$. Also, for a test particle at a distance $R$, the gravitational potential due to the Sun is $-G M / R$. Therefore, the total potential energy around the Sun, at a distance $R$ is given by,

$$
\phi(R)=-\frac{G M}{R}+4 \pi B_{0}^{2} R .
$$

The additional contribution to the Newtonian gravitational potential here is due to the modification in the gravitational field by the magnetic field energy density. The effect of the new potential term correspond to a constant acceleration depending on $B_{0}^{2}$. This corresponds to the extraperihelion precessions, such as that observed in the case of the Pioneer anomaly. However, the acceleration depends on the magnetic field energy density enclosed within an orbit, hence varies for different planetary orbits. Also, the induced velocity drag depends on the orbital speed of the planet as well (see also, Mirza 2019).

In Table 1 below, we give the shift $\Delta_{c a l}=d v$ in the velocity of the inner planetary orbits per planetary cycle, and compare it with the recent observational data (Iorio 1019; Iorio 2015; and references therein). The additional energy term above here corresponds to the dimensionless energy $d E / 2 E=d v / v$, where $v$ is the orbital speed of the planet.

\begin{tabular}{|l|l|l|l|l|l|}
\hline Planet & $\theta($ mas $/ \mathrm{cy})$ & $R(\mathrm{~km})$ & $v(\mathrm{~km} / \mathrm{s})$ & $\Delta_{\text {obs }}(\mathrm{km} / \mathrm{cycle})$ & $\Delta_{\text {cal }}(\mathrm{km} / \mathrm{cycle})$ \\
\hline Mercury & $-2.0 \pm 3.0$ & $58 \times 10^{6}$ & 47 & $-8.8336-1.7667$ & -3.5014 \\
\hline Venus & $2.6 \pm 1.6$ & $108 \times 10^{6}$ & 47 & $3.2898-13.8173$ & 5.7300 \\
\hline Earth & $0.19 \pm 0.19$ & $150 \times 10^{6}$ & 30 & $0-1.7362$ & 1.4881 \\
\hline Mars & $0.020 \pm 0.037$ & $228 \times 10^{6}$ & 24 & $-0.1180-0.5000$ & 0.0895 \\
\hline Jupiter & $58.2 \pm 28.3$ & $778 \times 10^{6}$ & 13 & $708.6071-2049.9838$ & 2270.74 \\
\hline Saturn & $0.15 \pm 0.65$ & $1.434 \times 10^{9}$ & 10 & $-21.8403-34.9451$ & 4.5918 \\
\hline
\end{tabular}


Table 1: The observed projected distance increase given by $\Delta_{\text {obs }}=2 \pi R \tan \left(\theta \times 10^{-3} / 3600\right)$, in $\mathrm{km}$ per planetary cycle (with Earth year $\approx 3.1536 \times 10^{7} \mathrm{~s}$ ), compared with the calculated shift $\Delta_{\text {cal }}$. The observed planetary secular perihelion precessions $\theta$ is in milliarcseconds per century (mas/cy), and the shifts are scaled per century. Here $R$ is the radial distance, and $v$ is orbital speed of the planet around the Sun.

It is notable here that, whereas Mercury, Venus, and Saturn have no intrinsic magnetic field, Earth, Mars, and Jupiter have locally generated equatorial fields of magnitude approximately $0.3 G, 0.007 G$, and $4 G$, respectively. This corresponds to a local velocity drag $\left(v_{\text {local }} \approx \sqrt{B_{\text {local }}^{2} R_{\text {planet }} / 2 \pi}\right)$, along the orbits of these planets, which is given by $0.3390 \mathrm{~km} / \mathrm{s}, 0.0194 \mathrm{~km} / \mathrm{s}$, and $51.7253 \mathrm{~km} / \mathrm{s}$, respectively. The local amplification induced in the solar velocity drag is taken into account in the calculated values of the shift $\Delta_{c a l}$ for the planets with significant magnetic field strengths. The calculated shift lies, within the range of observation errors, in the same range (per planetary cycle) as observed.

In equation (12) the first term on the rhs. decreases inversely as the distance, where as the second term increases linearly with increasing the disc radius $R$. The Newtonian gravitational effects therefore dominate the particle dynamics up to a distance $R_{0}$, such that $\phi\left(R_{0}\right)=0$. This corresponds to the minimum of the potential energy at $\phi\left(R_{0}\right)=0$, which gives for the effective range of the gravitational potential $R_{0}=\sqrt{G M / 4 \pi B_{0}^{2}}$. Putting the values of $M=M_{\odot}=$ $1.989 \times 10^{30} \mathrm{~kg}$, and $B_{0}=10 G=10^{-3} \mathrm{~T}$ for normal solar activity period, we obtain $R_{0}=31.82 \mathrm{AU}$. This is comparable to the semi-major axis of Neptune $(\approx$ $30.06 \mathrm{AU})$. Therefore, in the solar system, the Newtonian gravitational potential dominates the planetary dynamics up to Neptune (Fig. 1). The potential then changes sign and the repulsive potential due to the toroidal field becomes effective. For Pluto (semi-major axis $\approx 39.52 \mathrm{AU}$ ), the toroidal field energy exceeds by a factor of $1.609 \times 10^{6}$, whereas the drag velocity is approximately $0.22 \times 10^{-4} \mathrm{~km} / \mathrm{s}$. This value lies below the range of observational error as measured for the orbit of Pluto in the recent flyby mission New Horizon (Buie \& Folkner 2015). Also, for Uranus (19 AU) and Neptune (30 AU), the velocity change is less than $0.20 \times 10^{-4} \mathrm{~km} / \mathrm{s}$ and $0.1 \times 10^{-4} \mathrm{~km} / \mathrm{s}$, respectively, which also lie below the range of observational error (Iorio \& Giudice 2006).

The drag velocity has the first zero at $\varphi-\omega(R) t= \pm \pi / 2$, after which the source effects of the dragging on the test particle vanish. Neglecting the angular momentum due to the giant planets we have, at the radius $R=R_{\max }$ in the ecliptic, $J=1.1 \times 10^{42} \mathrm{kgm}^{2} / \mathrm{s}$. Along the axis $\varphi=0$, we have $R_{\max }^{3}=J t /(\pi / 2)$; which gives for an orbital period around the Sun, $t=2.592 \times 10^{6} \mathrm{~s}$. Hence, for the extent of the dragging effects we have the upper limit, approximately given by $R_{\max }=10^{5} \mathrm{AU}$. This region correspond to the Kuiper belt in the solar system. Here, as shown above, the repulsive toroidal field drag is greater than the attractive Newtonian gravitational potential. Also, for distances $R>$ $R_{\max }$, only attractive gravitational potential remains effective, since toroidal field effects reduce to zero at $R=R_{\max }$.

Using the field energy densities, we can also estimate the semi-major axis for a planet whose orbit transverses the Kuiper belt region $R_{0}<R<R_{\max }$. 
For an elliptical orbit, the semi-major axis $a$ of a test body is proportional to the total energy $E$. At points of extrema, the velocity vanishes (stationary points), therefore, the total energy equals the potential energy at aphelion $r_{a}$. Denoting the semi-major axis under the gravitational potential energy $E\left(r_{a}\right)=$ $G M\left(r_{a}\right) / r_{a}$ by $a_{g}$, and by $a_{\varphi}$ due to the toroidal field only, we have,

$$
\frac{a_{\varphi}}{a_{g}}=\frac{\left(4 \pi B_{0}^{2} r_{a}\right) V}{G M\left(r_{a}\right) / r_{a}}
$$

where $V=4 \pi r_{a}^{3} / 3$. Putting for the solar surface $r_{a} \approx R_{\odot}$, we obtain the scaling relation $a_{\varphi} \approx\left(10^{15} m\right) a_{g}$. Equation (13) implies that the semi-major axis in an orbit around the Sun increases by a factor of $10^{4} \mathrm{AU}$ under the toroidal field. This corresponds to the observed high eccentricity of KBOs. It therefore follows that outside the disc, with radius of approximately $31.82 \mathrm{AU}$, the outward dragging causes velocity increase in the planetary orbits, giving the highly eccentric periodic orbital motion observed for the KBOs.

\section{Conclusions and Summary}

In the above, we have shown that the coupled field dragging effect not only induces orbital changes in the inner planetary orbits, but has long range effects in the solar system, extending up to the Kuiper belt. For the KBOs, modification in the spacetime dragging causes the following observable features.

(1) The clustering of the KBOs in the solar ecliptic is due to the minimum of the outward spacetime drag which, according to equation (6), lies in the equatorial plane of the Sun (see also, Fig. 2).

(2) The minimum of the potential energy function is used to determine the distance up to which the Newtonian gravitational potential dominates the planetary motion. This gives for the range of the inner solar system the approximate distance up to Neptune. For distances larger than Neptune, toroidal field dragging plays a more effective role in the orbital dynamics than the Newtonian gravitational potential. This causes the formation of a Kuiper belt-like region.

(3) In the Kuiper belt, the coupled outward drag has maximum magnitude in the solar ecliptic, which allows KBOs to move through the ecliptic, with increased velocity and high eccentricity, in orbits of larger semi-major axes.

Summarizing, the effects of the spacetime dragging have been calculated here for the planetary orbits around the Sun. For the inner planets, the spacetime dragging induces a cyclic shift in the orbits. It was found that the derived magnitudes of the shift in the orbital velocity of the inner planets correspond to the observed shifts, lying within the range of observational accuracy.

We have also shown here that the orbital dynamics of KBOs is dominated by the toroidal field induced spacetime dragging. The coupling of the solar gravitational field to the solar toroidal magnetic field thus modifies the gravitational field of the Sun. This modification exceeds the Newtonian gravitational potential at sufficiently large distances. In contrast with the strong spacetime wrapping around rotating compact stars, the solar magnetic field generated 
dragging extends much farther in space for normal stars. For Sun-like stars, this is due to the weaker spacetime wrapping, since the lines of force (per unit volume) are relatively less closely bound around the star.

Acknowledgement

I gratefully acknowledge the anonymous reviewer for suggestions regarding recent planetary ephemerides.

\section{References}

[1] Batygin, K., et al. 2019, Physics Reports, 805, 1.

[2] Batygin, K., \& Brown, M. E. 2016a, AJ, 151, 22.

[3] Batygin, K., Brown, M.E. 2016b, ApJL. 833, L3.

[4] Batygin, K., Brown, M. E., \& Fraser, W. C. 2011, ApJ, 738, 13.

[5] Brown, M.E., Batygin, K. 2016, ApJL. 824, L23.

[6] Buie, M. W. and Folkner, W.N. 2015, AJ, 149, 22.

[7] Ciolfi, R. 2014, Astron. Nach. 335, 285.

[8] Debono, I. \& G.F. Smoot, G. F. 2016, Universe, 2, 23.

[9] Harding, A., Lai, D. 2006, Rep. Prog. Phys. 69, 2631.

[10] Iorio, L. 2019, AJ., 157, 220.

[11] Iorio, L. 2018, MNRAS. 476, 1811.

[12] Iorio, L. 2017, Astrophys. Space Sci. 326, 11.

[13] Iorio, L. 2015, Universe, 1, 38.

[14] Iorio, L. 2015, IJMPD 24, 1530015.

[15] Iorio, L. 2012, Solar Physics. 281, 815.

[16] Iorio, L. et al. 2011, Astrophys Space Sci. 331, 351.

[17] Iorio, L., Giudice, G. 2006, New Astronomy 11, 600.

[18] Lense, J., Thirring, H. 1918, Phys. Z. 19, 156. English translation in Mashhoon, B., Heh, F. W., \& Theiss, D. S. 1986, GRG 16, 711.

[19] Levison, H. F., Morbidelli, A., Van Laerhoven, C., Gomes, R., \& Tsiganis, K. 2008, Icar, 196, 258.

[20] Lucchesi, D. et al. 2019, Universe, 5, 141.

[21] Makishima, K. et al. 2014, Phys. Rev. Lett. 112, 171102. 
[22] Madigan, A.-M., \& McCourt, M. 2015, arXiv:1509.08920,

[23] Marcos, C. de la F. \& Marcos, R. de la F. 2014, MNRAS, 443, L59.

[24] Mereghetti, S. 2008, Astron. Astrophys. Rev. 15, 225.

[25] Mirza, B. M. 2019, MNRAS 489, 3232.

[26] Mirza, B. M. 2017, ApJ. 847, 73.

[27] Mirza, B. M. 2007, IJMPD, 16, 1705.

[28] Mitchell, J. P. et al. 2015, MNRAS, 447, 1213.

[29] Muhlberger C. D. et al. 2014, Phys. Rev. D 90, 104014.

[30] Nottale, L. 2003, arXiv preprint gr-qc/0307042

[31] Oron, A. 2002, Physical Review D 66, 023006.

[32] Petit, P., et al. 2008, MNRAS 388, 80.

[33] Pétri, J., 2013, MNRAS 433, 986.

[34] Renzetti, G. 2013, Open Physics 11, 531.

[35] Rindler, W. 1997, Phys. Lett. A 233, 25.

[36] Tartaglia, A. \& Ruggiero, M. L. 2002, Il Nuovo Cimento B, 117, 743.

[37] Thompson, C., and Duncan, R. C. 1995, MNRAS. 275, 255.

[38] Tiengo, A., et al. 2013, Natur, 500, 312.

[39] Trujillo, C. A., \& Sheppard, S. S. 2014, Natur, 507, 471.

Figure Captions:

Figure 1: The total potential energy function (12) per unit gravitational potential energy (G.P.E.) of a planet. The gravitational potential dominates approximately up to the orbit of Neptune. Here the corresponding Kuiper belt region extends to the radius between $31.82 \mathrm{AU}$ to $10^{5} \mathrm{AU}$, where the toroidal potential is effectively higher than the Newtonian gravitational potential.

Figure 2: Orbital clustering in the ecliptic due to the local minima of the toroidal potential in the plane $\theta=\pi / 2$. The potential is maximum at the poles, causing the KBOs to group towards the ecliptic plane, particularly while crossing the Kuiper belt where the toroidal potential energy exceeds the Newtonian gravitational potential. 
This figure "Figure_1.png" is available in "png" format from: http://arxiv.org/ps/2002.12774v1 
This figure "Figure_2_2_.png" is available in "png" format from: http://arxiv.org/ps/2002.12774v1 